\title{
One pot light assisted green synthesis, storage and antimicrobial activity of dextran stabilized silver nanoparticles
}

\author{
Muhammad Ajaz Hussain ${ }^{1 *}$, Abdullah Shah ${ }^{1}$, Ibrahim Jantan², Muhammad Nawaz Tahir ${ }^{3}$, Muhammad Raza Shah ${ }^{4}$, \\ Riaz Ahmed ${ }^{5}$ and Syed Nasir Abbas Bukhari ${ }^{*}$
}

\begin{abstract}
Background: Green synthesis of nanomaterials finds the edge over chemical methods due to its environmental compatibility. Herein, we report green synthesis of silver nanoparticles (Ag NPs) mediated with dextran. Dextran was used as a stabilizer and capping agent to synthesize Ag NPs using silver nitrate $\left(\mathrm{AgNO}_{3}\right)$ under diffused sunlight conditions.

Results: UV-vis spectra of as synthesized Ag nanoparticles showed characteristic surface plasmon band in the range from $\sim 405-452 \mathrm{~nm}$. Scanning electron microscopy (SEM) and atomic force microscopy (AFM) studies showed spherical Ag NPs in the size regime of $\sim 50-70 \mathrm{~nm}$. Face centered cubic lattice of Ag NPs was confirmed by powder X-ray diffraction (PXRD). FT-IR spectroscopy confirmed that dextran not only acts as reducing agent but also functionalizes the surfaces of Ag NPs to make very stable dispersions. Moreover, on drying, the solution of dextran stabilized Ag NPs resulted in the formation of thin films which were found stable over months with no change in the plasmon band of pristine Ag NPs. The antimicrobial assay of the as synthesized Ag NPs showed remarkable activity.
\end{abstract}

Conclusion: Being significantly active against microbes, the Ag NPs can be explored for antimicrobial medical devices.

Keywords: Ag nanoparticles, Storage of nanoparticles, Diffused sun light, Antimicrobial activity

\section{Introduction}

Ag NPs have wide variety of applications, e.g., optoelectrical [1,2], microbiocidal [3], nanorobotics [4] and medicinal [5]. However, clustering of Ag NPs on storage and in physiological media [6-9] is a major limitation in their biomedical applications. Chemical methods for the synthesis of Ag NPs have harmful effects on environment as well as on human health [9]. Due to said reasons, nowadays, polysaccharides and polypeptides [10] have attracted the vigil eye of researchers for the biosynthesis of Ag NPs as they can act as reducing, capping and stabilizing agents [11-13]. Recently, polysaccharide based Ag NPs have been prepared by adding $\mathrm{NaOH}$ [14] but use of such corrosive reagent has harmful effects on

\footnotetext{
* Correspondence: majaz172@yahoo.com; snab@ukm.edu.my 'Department of Chemistry, University of Sargodha, Sargodha 40100, Pakistan ${ }^{2}$ Drug and Herbal Research Centre, Faculty of Pharmacy, Universiti Kebangsaan Malaysia, Jalan Raja Muda Abdul Aziz, Kuala Lumpur 50300, Malaysia

Full list of author information is available at the end of the article
}

environment as well as on human health. Therefore, it is promising to fabricate Ag NPs that could sustain themselves for longer period of time using environmentally benign molecules like biopolymers.

In this report, we have explored the green synthesis of Ag NPs using dextran as co-reducing as well as capping ligand without using any environmentally hostile ingredient like $\mathrm{NaOH}$ and $\mathrm{NaBH}_{4}$. Dextran was choice because it is cheaper, non-toxic, biocompatible, efficient reducing and self-capping agent, in situ stabilizer of nanoparticles and environment friendly. The as synthesized NPs can be stored within matrix of dextran in the form of thin films without changing the optical properties over months. Moreover, the as-prepared Ag NPs were tested as antimicrobial probes against $S$. aureus (ATCC 25923), E. coli (ATCC 25922), B. subtilis (ATCC 6633), S. epidermidis (ATCC 12228), P. aeruginosa (ATCC 27853) and fungal strains Actinomycetes and A. niger. 

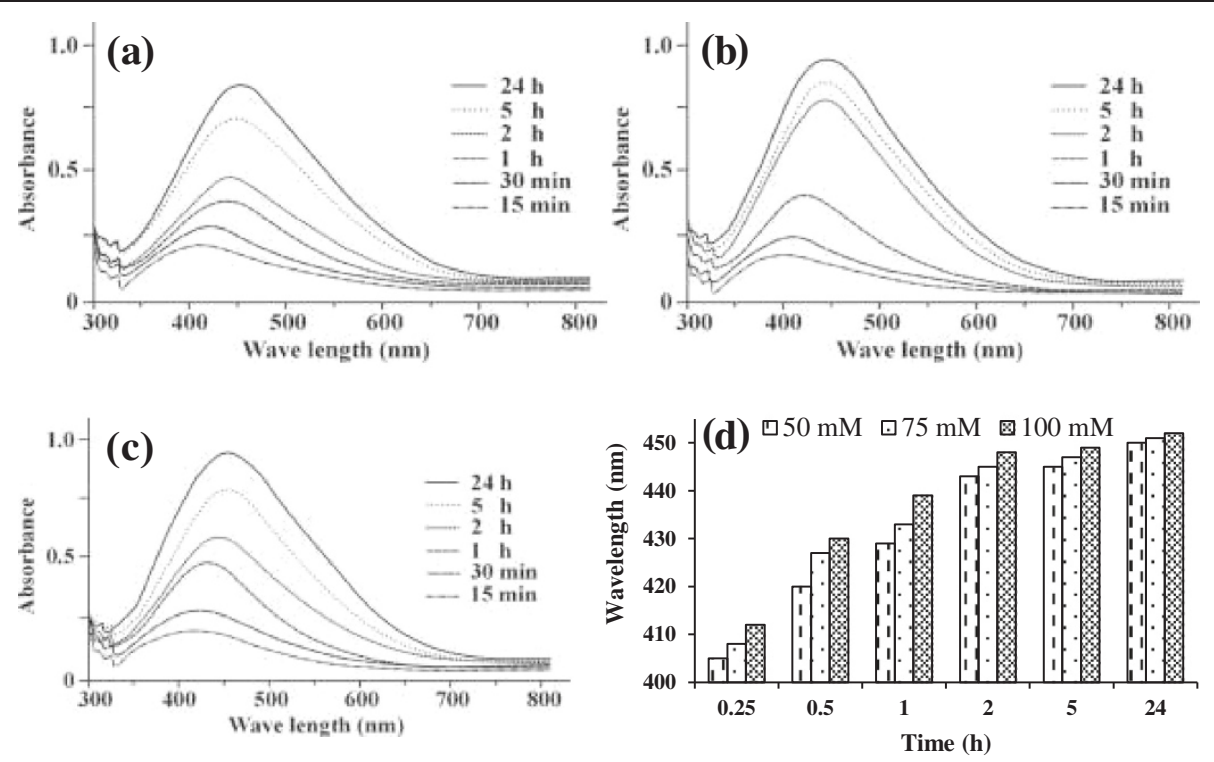

Figure 1 UV-vis spectra of Ag NPs prepared in dextran: 50 (a), 75 (b) and $100 \mathrm{mmol}$ (c) and graph showing effect of reaction time and concentration on absorbance (d).

\section{Experimental}

\section{Materials and measurements}

Dextran (molar mass 40000) was obtained from Sigma Aldrich, Germany. $\mathrm{AgNO}_{3}$ (99.98\%) from Merck, Germany was used as silver precursor. Deionized water was used for preparation of all solutions. UV-vis analyses were performed on UV-1700 PharmaSpec (Shimadzu, Japan). FT-IR spectra were recorded on IR Prestige-21 (Shimadzu, Japan). The samples (microtomes) were analyzed by SEM Plano (Wetzlar, Germany) using carbon stubs (carbon adhesive Leit-Tabs No. G 3347). The sizes and shapes of NPs were analyzed using AFM, Multimode, Nanoscope IIIa, Veeco, (California, USA) in tapping mode. Powder $\mathrm{X}$-ray diffraction measurements were carried out (over a range of $5-100^{\circ}, 2 \Theta$ ) on an Xpert Pro MPD, (PANalytical, The Netherlands) diffractometer equipped with monochromatic X-rays.

\section{Sample preparation of $\mathrm{AgNO}_{3}$ and dextran}

$\mathrm{AgNO}_{3}$ solutions $(50,75$ and $100 \mathrm{mmol}$ ) were prepared by dissolving $\mathrm{AgNO}_{3}(0.85,1.27$ and $1.7 \mathrm{~g}$, respectively) in deionized water. Concentrated solution of dextran was freshly prepared by dissolving dextran in deionized water $(10 \mathrm{~mL})$.

\section{Synthesis of Ag NPs mediated by dextran}

Freshly prepared $\mathrm{AgNO}_{3}(50 \mathrm{mmol}, 2 \mathrm{~mL})$ solution was added to the dextran solution $(2 \mathrm{~mL})$. The reaction mixture was exposed to diffused sunlight and color change was monitored over a period of $24 \mathrm{~h}$ by using UV-vis spectrophotometer. The same procedure was adopted for $\mathrm{AgNO}_{3}$ (75 and $100 \mathrm{mmol}$ ) solutions, respectively.
Thin film formation of dextran loaded with Ag NPs

Concentrated aq. solution of dextran loaded with Ag NPs $(100 \mathrm{mmol})$ was kept in a petri dish for drying under air and stored.

\section{Atomic force microscopy (AFM)}

The samples were prepared by dissolving thin films in deionized water and dispersing them on freshly cleaved sheet of mica substrate. AFM images were recorded at ambient temperature and repeated with different concentrations of the samples.

\section{Scanning electron microscope (SEM)}

Surface of dextran thin films was analyzed by SEM to study geometry of embedded Ag NPs.

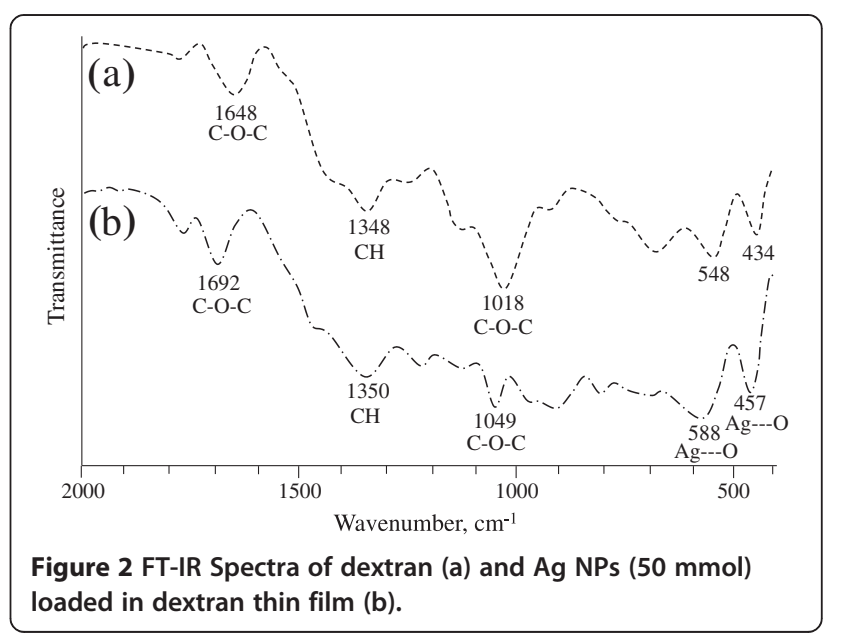





Figure 3 SEM images of Ag NPs (50-70 nm) embedded in dextran thin films of 50 (a), 75 (b) and 100 mmol (c) AgNO $_{3}$ solution.

\section{Antimicrobial activity of Ag NPs}

The test organism $S$. aureus (ATCC 25923), E. coli (ATCC 25922), B. subtilis (ATCC 6633), S. epidermidis (ATCC 12228), $P$. aeruginosa (ATCC 27853) and fungal strains Actinomycetes and A. niger were used for testing the antimicrobial activity of Ag NPs. The bacterial and fungal strains were procured from Microbiology Labs of Agriculture University, Faisalabad, Pakistan. Mueller Hinton Agar Media (Oxoid Ltd., England) was used for bacterial growth and Sabouraud Dextrose Agar (Hardy Diagnostics, USA) was used for fungal growth. Inoculums were prepared by transferring the microorganism culture in both tubes having $10 \mathrm{~mL}$ of respective broth media (Mueller Hinton broth for bacterial culture and Sabouraud Dextrose broth for fungal culture) and were inoculated for $24 \mathrm{~h}$ at $37^{\circ} \mathrm{C}$ for bacteria and $27-30^{\circ} \mathrm{C}$ for fungi. Seven days old culture of fungal strain was washed and suspended in normal saline solution. Then filtered through glass wool aseptically and incubated at $28^{\circ} \mathrm{C}$. The tubes were shaken periodically to accelerate the growth of microorganisms. The turbidity of inoculums was adjusted by $0.5 \mathrm{Mc}$ Farland Standard.

Antimicrobial assay of Ag NPs against different bacterial and fungal strains was conducted by disc diffusion method. In vitro antimicrobial activity was screened by using Mueller Hinton Agar plates for bacterial strains. Inoculum $(0.1 \mathrm{~mL})$ was spread uniformly on plates. Ag NPs solution was loaded on $6 \mathrm{~mm}$ discs of Whatman No. 1 filter paper. Loaded discs were placed on the surface of medium and plates were incubated for $24 \mathrm{~h}$ at $37^{\circ} \mathrm{C}$. Pure DMSO $(15-20 \mathrm{~mL})$ loaded disc was used as negative control. At the end of incubation period, inhibition zones were measured in millimeters. These studies were performed in triplicate.

Similarly, antifungal activity of Ag NPs was screened on Sabouraud Dextrose Agar plates by using disc diffusion method and plates were incubated at $27-30^{\circ} \mathrm{C}$ for 36-48 h. After incubation period, zones of inhibition were measured.

\section{Results and Discussion}

$\mathrm{AgNO}_{3}$ (50, 75 and $100 \mathrm{mmol}$ ) solutions mixed with concentrated dextran solution were colorless in the beginning but turned light brown after 10 min indicating the nucleation of Ag NPs. The color changed to ruby red after $60 \mathrm{~min}$ while chocolate red color was observed after $24 \mathrm{~h}$ indicating the completion of growth process.

UV-vis absorption bands appeared ranging from $~ 405-$ 450, 408-451 and 412-452 nm for nanoparticles synthesized using 50, 75 and $100 \mathrm{mmol}$ dextran- $\mathrm{AgNO}_{3}$ solutions, respectively and the corresponding UV-vis spectra of dextran-Ag NPs are shown in Figure 1a,b and c. The all reactions were monitored for $24 \mathrm{~h}$ at different time intervals. The red shift was observed in UV-vis absorptions for dextran-Ag NPs by increasing reaction time. Increase in absorption coefficient was also observed by increasing the concentration of $\mathrm{AgNO}_{3}$ solution from 50-100 mmol. The increase in wavelength of absorption may be attributed to increase in size of Ag NPs. It is noteworthy that no absorption band was observed in the spectrum when sample was stored in dark. The reaction progressed on exposing the sample to diffused sunlight. Graphical representation of increase in absorption of $\mathrm{Ag}$ NPs solutions with increase in reaction time and $\mathrm{AgNO}_{3}$ concentration (50, 75 and $100 \mathrm{mmol}$ ) is depicted in Figure 1d.

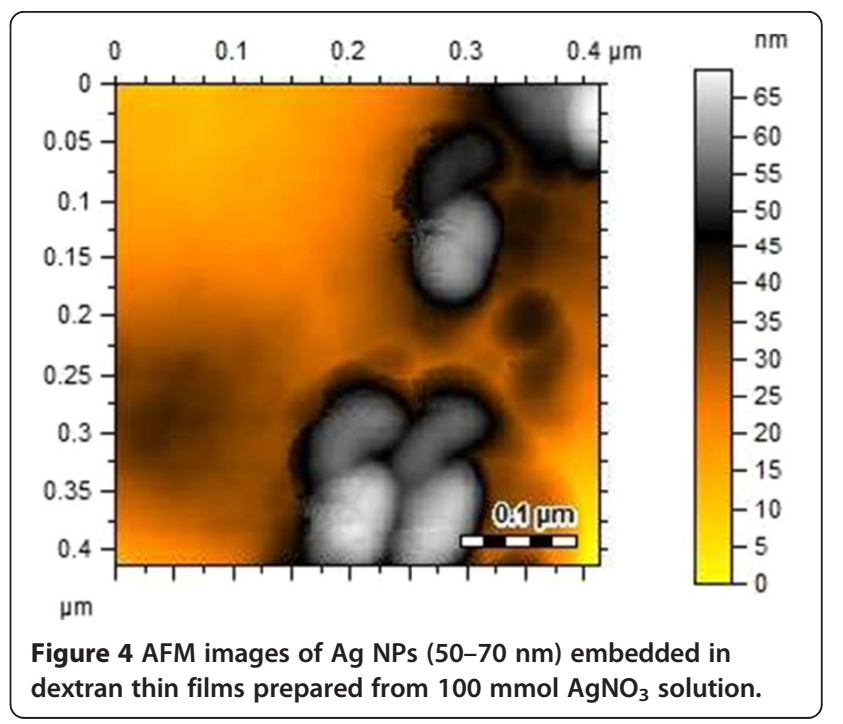




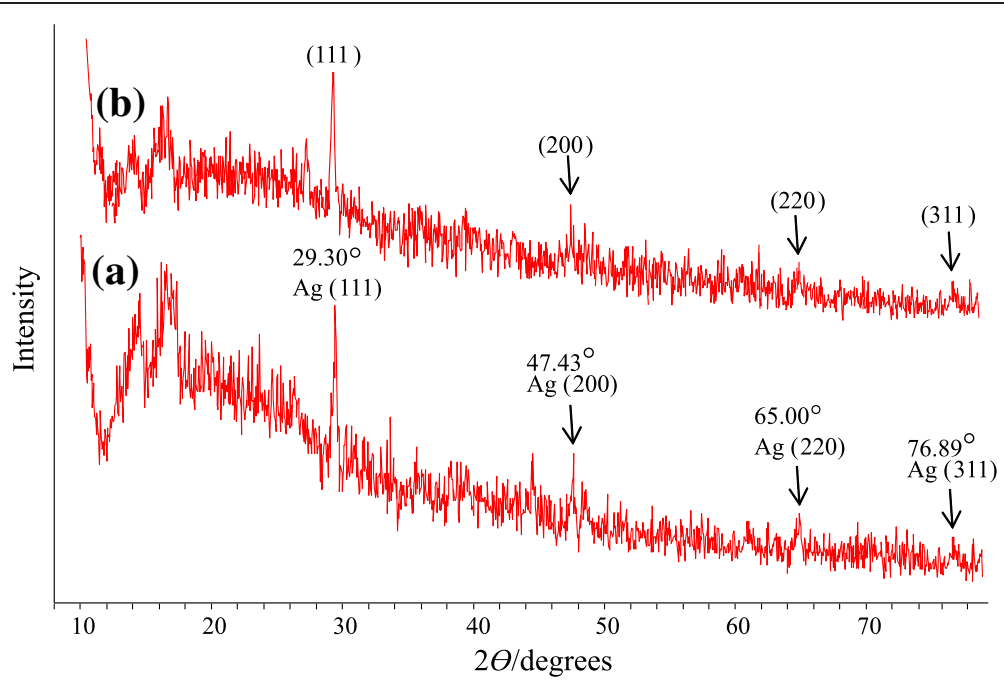

Figure 5 PXRD Spectra of dextran-Ag NPs (100 mmol); (a) fresh sample and (b) recorded after one year storage.

FT-IR (using pellet mixed with $\mathrm{KBr}$ ) spectra of dextran and dextran-Ag NPs (50 mmol solution) were recorded to confirm interaction between dextran and $\mathrm{Ag}^{+}$ions. Peaks at 434 and $548 \mathrm{~cm}^{-1}$ in pure dextran were shifted to 457 and $588 \mathrm{~cm}^{-1}$ in dextran-Ag NPs due to Ag--O excitation [15]. It is obvious from FT-IR spectra that there exist significant Van der Waal interactions between the chain of dextran and Ag NPs as all of the signals of dextran were shifted to somewhat higher positions (Figure 2).

Microtomes of dextran thin films loaded with Ag NPs observed by SEM showed spherical Ag NPs with uniform distribution (Figure 3). Dextran-Ag NPs film was dissolved in Milli-Q water and studied by AFM as well. The AFM images also witnessed the results of SEM that the NPs were found spherical (50-70 nm, Figure 4).

The crystalline nature of the as synthesized Ag NPs using dextran was confirmed via XRD analysis. As shown in Figure 5, there are four distinct reflections in the diffractogram at $29.3^{\circ}(111), 47.43^{\circ}$ (200), $65.05^{\circ}$ (220), and $76.89^{\circ}(311)$. These characteristics reflections show crystallographic planes of face centered cubic structure of the Ag NPs. Same sample was stored in the form of thin film for one year and PXRD was rerecorded to confirm the structural stability of the Ag NPs. Similarity of PXRD pattern in sample before and after one year (Figure 4) indicated that Ag NPs are quite stable on storage in thin film of dextran. In this way we could successfully avoid agglomeration of Ag NPs on storage in solid state. So, this novel method for long term storage of Ag NPs can be further exploited for potential biomedical applications and optoelectronic devices.

There was no difference in SEM images of the sample (100 mmol) before and after one year storage (Figure 6) in thin films. The synthesized thin films were foldable, ruby red in color and almost optically transparent as demonstrated by digital photograph (see Figure 6). Likewise, after one year storage of thin films under dark were re-dissolved in water. The UV-vis spectroscopic analysis of aqueous solution $(100 \mathrm{mmol})$ of thin films (see Figure 6) after one year showed an absorption band centered at $446 \mathrm{~nm}$. The concordant absorbance therefore indicated no change in size and morphology of the stored Ag NPs



Figure 6 SEM image and UV-vis spectrum of Ag NPs (100 mmol, $5 \mathrm{~h}$ reaction time) embedded in dextran thin films (foldable and see through) after one year storage; vial is indicating the color of stored thin films after dissolution in water. 

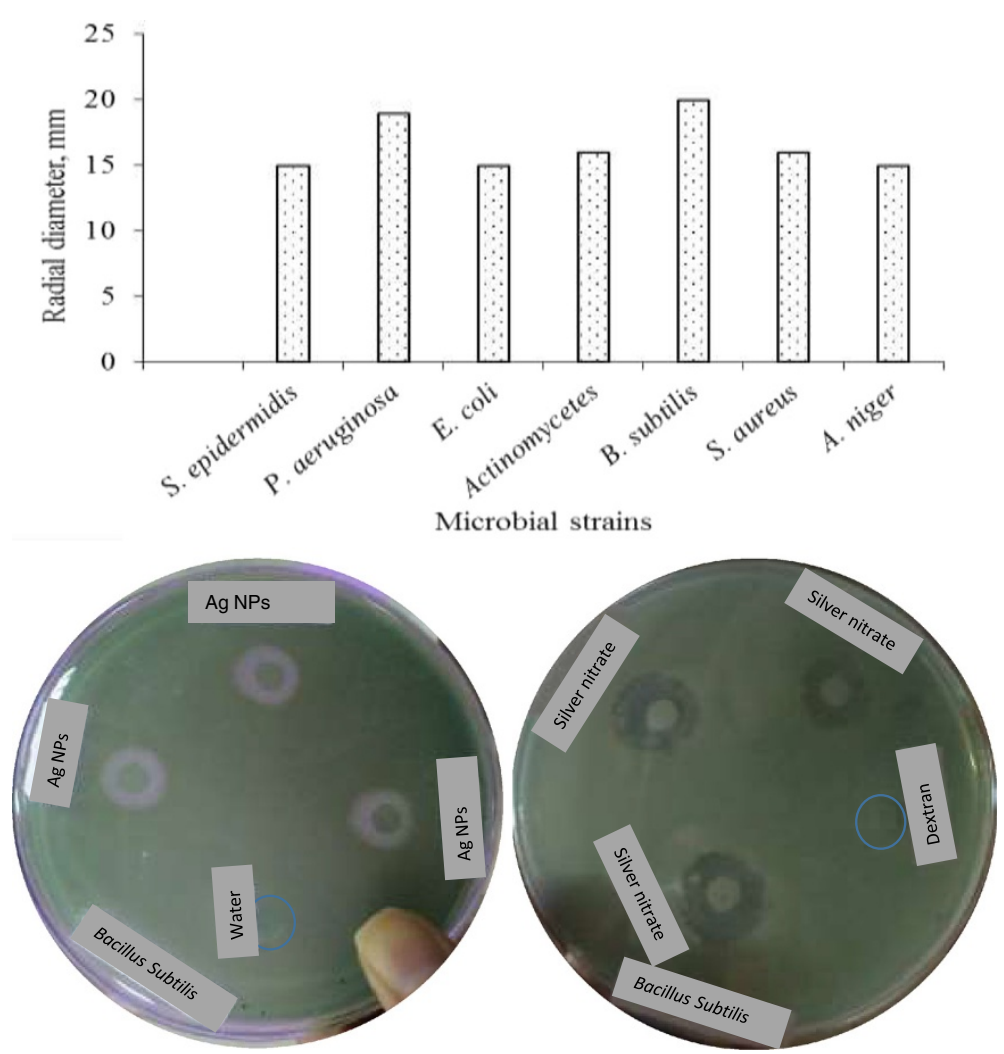

Figure $7 \mathrm{Graph}$ indicating inhibitory zone (radial diameter) of Ag NPs $(50 \mathrm{mmol})$ vs. different microbial strains whereas plates indicating that deionized water and dextran did not show any activity however, $\mathrm{AgNO}_{3}$ solution $(0.01 \mathrm{M})$ was found active against Bacillus subtilis strains.

in thin films. So, the present method appeared highly efficient for the long term storage of Ag NPs in dextran thin films without agglomeration.

Solution of Ag NPs showed significant antimicrobial activity against different bacterial (S. aureus, E. coli, B. subtilis, S. epidermidis, P. aeruginosa) and fungal strains Actinomycetes and A. niger as depicted in Figure 7. The inhibiting zones vs. microbial strains for Ag NPs solution of $50 \mathrm{mmol}$ concentration and antibacterial activity of silver nanoparticles (Ag NPs) against Bacillus subtilis are also shown as a typical example (see Figure 7). It was observed that deionized water and dextran do not show any activity however, $\mathrm{AgNO}_{3}$ solution $(0.01 \mathrm{M})$ was found active against mentioned strains. All of the experiments were carried out in triplicate and mean values have been reported. The prepared pristine Ag NPs can be used as effective therapeutic tools.

\section{Conclusions}

We report on the diffused sun light assisted green synthesis of dextran stabilized Ag NPs without use of any hazardous and costly reducing agent or any extra functionalizing ligand. The as synthesized nanoparticles can be stored in solid state over months without imparting any change in the physical or optical properties. Being significantly active against microbes, the Ag NPs can be exploited for antimicrobial medical devices.

\section{Competing interests}

The authors declare that they have no competing interests.

\section{Authors' contributions}

MAH contributed in conception, design and acquisition of data. MAH and IJ have given final approval of the version to be published. AS synthesized the Ag nanoparticles and performed UV spectroscopic analysis. IJ and MNT contributed in spectral analysis and interpretation of experimental data. MRS performed and interpreted the AFM analysis. RA performed SEM and critical revision of scientific contents. SNAB performed antimicrobial analysis and involved in revising the manuscript critically for important intellectual content. All authors read and approved the final manuscript.

\section{Author details}

'Department of Chemistry, University of Sargodha, Sargodha 40100, Pakistan. ${ }^{2}$ Drug and Herbal Research Centre, Faculty of Pharmacy, Universiti Kebangsaan Malaysia, Jalan Raja Muda Abdul Aziz, Kuala Lumpur 50300, Malaysia. Institute of Inorganic and Analytical Chemistry, Johannes Guttenberg University of Mainz, Duesbergweg 10-14, Mainz 55128, Germany. ${ }^{4}$ International Center for Chemical and Biological Sciences, University of Karachi, Karachi 75270, Pakistan. ${ }^{5}$ Centre for Advanced Studies in Physics (CASP), GC University, Lahore 54000, Pakistan. 
Received: 15 October 2014 Accepted: 14 November 2014

Published online: 03 December 2014

\section{References}

1. Raveendran P, Fu J, Wallen SL: Completely "green" synthesis and stabilization of metal nanoparticles. J Am Chem Soc 2003, 125:13940-13941.

2. Carsin H, Wassermann D, Pannier M, Dumas R, Bohbot S: A silver sulphadiazine-impregnated lipidocolloid wound dressing to treat second-degree burns. J Wound Care 2004, 13:145-148.

3. Hayward RC, Saville DA, Aksay IA: Electrophoretic assembly of colloidal crystals with optically tunable micropatterns. Nature 2000, 404:56-59.

4. Haberzettl CA: Nanomedicine: destination or journey. Nanotechnol 2002, 13:9-13.

5. Ong C, Lim JZZ, Ng C-T, Li JJ, Yung L-YL, Bay B-H: Silver nanoparticles in cancer: therapeutic efficacy and toxicity. Curr Med Chem 2013, 20:772-781.

6. El-Nour KMMA, Eftaiha A, Al-Warthan AA, Ammar RAA: Synthesis and applications of silver nanoparticles. Arab J Chem 2010, 3:135-140.

7. Tahir MN, Eberhardt M, Zink N, Therese HA, Kolb U, Theato P, Tremel W: From single molecules to nanoscopically structured functional materials: au nanocrystal growth on $\mathrm{TiO} 2$ nanowires controlled by surface-bound silicatein. Angew Chem Int Ed 2006, 45:4803-4809.

8. Tahir MN, Zink N, Eberhardt M, Therese HA, Kolb U, Faiss S, Janshoff A, Kolb $U$, Theato P, Tremel W: Hierarchical assembly of TiO2 nanoparticles on WS2 nanotubes achieved through multifunctional polymeric ligands. Small 2007, 3:829-834.

9. Tahir MN, Andre R, Sahoo JK, Jochum FD, Theato P, Natalio F, Berger R, Branscheid R, Kolb U, Tremel W: Hydrogen peroxide sensors for cellular imaging based on horse radish peroxidase reconstituted on polymer-functionalized TiO(2) nanorods. Nanoscale 2011, 3:3907-3914.

10. Bar H, Bhui DK, Sahoo GP, Sarkar P, De SP, Misra A: Green synthesis of silver nanoparticles using latex of Jatropha curcus. Colloid Surface A 2009, 339(1-3):134-139.

11. Lou C-W, Chen A-P, Lic T-T, Lin J-H: Antimicrobial activity of UV-induced chitosan capped silver nanoparticles. Mater Lett 2014, 128:248-252.

12. Oluwafemi OS, Vuyelwa N, Scriba M, Songca SP: Green controlled synthesis of monodispersed, stable and smaller sized starch-capped silver nanoparticles. Mater Lett 2013, 106:332-336.

13. Long Y, Ran X, Zhang L, Guo Q, Yang T, Gao J, Cheng H, Cheng T, Shi C, Su Y: A method for the preparation of silver nanoparticles using commercially available carboxymethyl chitosan and sunlight. Mater Lett 2013, 112:101-104.

14. Bankura KP, Maity D, Mollick MMR, Mondal D, Bhowmick B, Bain MK, Chakraborty A, Sarkar J, Acharya K, Chattopadhyay D: Synthesis, characterization and antimicrobial activity of dextran stabilized silver nanoparticles in aqueous medium. Carohydr Polym 2012, 89:1159-1165.

15. Shameli $K$, Ahmad MB, Jazayeri SD, Sedaghat $S$, Shabanzadeh $P$, Jahangirian $\mathrm{H}$, Mahdavi M, Abdollahi Y: Synthesis and characterization of polyethylene glycol mediated silver nanoparticles by the green method. Int J Mol Sci 2012, 13:6639-6650

\section{doi:10.1186/s12951-014-0053-5}

Cite this article as: Hussain et al:: One pot light assisted green synthesis, storage and antimicrobial activity of dextran stabilized silver nanoparticles. Journal of Nanobiotechnology 2014 12:53.

\section{Submit your next manuscript to BioMed Central and take full advantage of:}

- Convenient online submission

- Thorough peer review

- No space constraints or color figure charges

- Immediate publication on acceptance

- Inclusion in PubMed, CAS, Scopus and Google Scholar

- Research which is freely available for redistribution 